\title{
NEUROLEPTIC MALIGNANT SYNDROME INDUCED BY ARIPIPRAZOLE - A CASE REPORT
}

\author{
Nayana Naik ${ }^{1}$, Yvonne Da Silva Pereira ${ }^{2}$, Ashish Srivastava ${ }^{3}$
}

\section{HOW TO CITE THIS ARTICLE:}

Nayana Naik, Yvonne Da Silva Pereira, Ashish Srivastava. "Neuroleptic malignant syndrome induced by aripiprazole - a case report". Journal of Evolution of Medical and Dental Sciences 2013; Vol2, Issue 33, August 19; Page: 6247-6250.

ABSTRACT: Neuroleptic malignant syndrome [NMS], although rare, is a well-documented lifethreatening reaction to antipsychotic medications with mortality rate estimated as being up to $20 \%$. NMS was traditionally attributed to potent dopamine antagonism of typical antipsychotics but cases of NMS have been reported for each of the newer atypical antipsychotics. Aripiprazole is one such atypical agent approved by FDA for treating schizophrenia in 2002 and acute bipolar mania in 2004. Aripiprazole is a dopamine D2 receptor partial agonist with partial agonist activity at 5- $\mathrm{HT}_{1 \mathrm{~A}}$ receptor and antagonist activity at $5-\mathrm{HT}_{2 \mathrm{~A}}$ receptor. In comparison to other atypical it posses unique mechanism of action that may limit development of hypodopaminergic state, however there are case reports of aripiprazole induced NMS, akathisia, rhabdomyolysis, parkinsonism and excessive somnolence in children. To add to this literature we report a case of 50 year old woman with multiple risk factors, developed NMS with low dose of aripiprazole.

INTRODUCTION: The neuroleptic malignant syndrome (NMS) is an idiopathic, life-threatening reaction to Antipsychotic medication, characterized principally by fever, muscle rigidity, altered consciousness, autonomic instability, laboratory findings such as elevated creatine phosphokinase (CPK), ${ }^{1}$ leukocytosis, raised liver enzymes. Serious complications are possible, including renal failure, thromboembolism, respiratory failure from chest wall rigidity, aspiration pneumonia, and arrhythmia ${ }^{2}$. Different criteria's are proposed by different researchers for diagnosis of NMS but most commonly used are DSM-IV TR, ${ }^{3}$ and Levensons, ${ }^{1}$ criteria. Treatment consists of immediate discontinuation of the antipsychotics as well as D2 blocking agents, and most commonly used pharmacologic interventions are bromocriptine, and dantrolene. ${ }^{4}$ we report a case of 50 year old lady with multiple risk factors, developed NMS with low dose of single agent, aripiprazole.

CASE REPORT: Ms XY 50 yrs old lady initially presented to the hospital in November 2009 and was diagnosed to be suffering from undifferentiated schizophrenia and was treated with olanzapine $5 \mathrm{mg}$ /day. Later during the course of illness she exhibited depressive symptoms and fluoxetine 20 $\mathrm{mg} /$ day was added which was stopped after 6 months as her depressive symptoms remitted. Olanzapine was discontinued after 2 yrs of treatment as she developed Diabetes Mellitus. Later she was initiated on Trifluoperazine $15 \mathrm{mg}$ daily which had to be stopped within two weeks due to severe extra pyramidal symptoms [EPS]. Trihexyphenidyl, $2 \mathrm{mg}$ /day was given for 4 weeks till her EPS subsided completely and tablet aripiprazole $2.5 \mathrm{mg} /$ day was started which was slowly increased to $5 \mathrm{mg}$ /day over next 4 weeks.

Within 4 weeks of starting with aripiprazole she developed EPS and at times she experienced acute dystonia. Trihexyphenidyl $2 \mathrm{mg}$ /day was restarted in view of this but low grade of EPS 
persisted. It was also noticed that she was getting frequent urinary tract infection which was treated each time as per urine culture and sensitivity test in consultation with Nephrologists. She would at time show catatonic features which would ameliorate within couple of days with addition of oral Lorazepam 1-2 mg per day. She was investigated on couple of occasion for NMS with CPK levels and blood total counts, both of which were normal. She took aripiprazole $5 \mathrm{mg}$ daily till 12/10/2012 but within next two days it was increased to $10 \mathrm{mg} /$ day in view of her relapse of psychotic symptoms. The very next day there was an abrupt change in her mental state and worsening of her extra pyramidal symptoms.

On examination she was confused, exhibited marked rigidity in addition to her extra pyramidal symptoms. She was mute and there was drooling of saliva, mild dehydration was noted. Her temperature was $101.4^{\circ} \mathrm{F}$ and pulse was 104 per minute, respiratory breath rate was $20 / \mathrm{min}$. Her blood pressure was $110 / 70 \mathrm{~mm} \mathrm{Hg}$ and no fluctuations were recorded. She was uncomfortable and was in moderate distress.

Laboratory findings include CPK level $2855 \mathrm{IU} / \mathrm{L}, \mathrm{Hb} 11 \mathrm{gm} / \mathrm{dl}$, total leucocytes 10500/mm³. Her urea, creatinine, serum electrolytes, and liver function tests were within normal limits. Her urine analysis showed numerous pus cells. Abdominal ultrasound showed thick urinary bladder wall.

Her brain tomography demonstrated mild atrophic change. Plain MRI of the brain revealed sub centric hyper intensities in both frontal and occipital white matter, corona radiate and in periventricular region, suggestive of non-specific etiology. Rest of the brain parenchyma was normal. No acute vascular event was reported on consultation with radiologist. Her ECG was unremarkable. From the patient's medical history and presentation, the diagnosis of NMS was made based on DSM-IV TR and Levensons criteria for diagnosing NMS. Ms XY received IV hydration, Paracetamol oral medication. Her aripiprazole was stopped immediately and she was started on bromocriptine $1.25 \mathrm{mg} /$ day, clonazepam $2 \mathrm{mg} /$ day. Her trihexyphenidyl was continued as 2 $\mathrm{mg} /$ day. Also she was started on antibiotics for urinary tract infection [UTI] along with urine alkalinizer awaiting urine culture and sensitivity report. She was treated in psychiatric unit in liaison with general medicine department.

Next day her temperature recorded was normal and she started to improve, aside from mild EPS. Her recovery was uneventful. Her CPK level was $25 \mathrm{IU} / \mathrm{L}$ within a week. She continued same dose of bromocriptine for 10 days and clonazepam was reduced and stopped over 4 weeks along with trihexyphenidyl.

She did not exhibit any psychotic features until a month and remained hospitalized for supervision of re-emergence of psychotic feature. Later she began developing catatonic symptoms hence she was been started on olanzapine $2.5 \mathrm{mg}$ /day and Lorazepam $2 \mathrm{mg} /$ day and thereafter has been maintaining fairly well without much symptoms.

DISCUSSION: Atypical neuroleptic agents have been shown to be highly effective and in general, safe, obtaining widespread use in medicine. However, it is imperative to note that all antipsychotics have been reported as having the ability to induce NMS, including rare reports of NMS caused by clozapine, olanzapine, and risperidone. ${ }^{5}$ This case presents occurrence NMS induced by one of the newest atypical antipsychotic, aripiprazole. 
NMS is an idiosyncratic reaction and cannot be predicted, but there are some identified risk factors. Young age, male gender, dehydration, agitation, rapid dose escalation, and intra-muscular administration increase the risk. 2, 6 Prior history of NMS increases the risk for future episodes. There is some evidence for an association between NMS and concurrent lithium treatment, poorly controlled extra pyramidal symptoms, patients with affective disorders, iron deficiency, poor nutrition, environmental heat load, catatonia, and those drugs that are more potent dopamine- 2 (D2) antagonists.4, 7

Our patient had majority of risk factor like rapid escalation of the dose, associated UTI, mild persisting EPS, atrophic brain changes, dehydration, all these factors may have contributed to development of NMS although she was receiving single agent small dose of aripiprazole which is considered comparatively safer than other atypical antipsychotics. In comparison to the other atypical, it possesses a unique mechanism of action that may limit the development of hypo dopaminergic states. Determining neuroleptic malignant syndrome risk with aripiprazole is difficult; two cases were reported in the premarketing sample. ${ }^{8}$ one animal study showed diminished catalepsy with chronic aripiprazole use, in contrast to persistent catalepsy with haloperidol. ${ }^{9}$ Adverse effects are probably underrepresented. Clinical deterioration and adverse effects were reported after starting, switching to, or combining aripiprazole with other antipsychotics or serotonergic agents (Trazodone, sertraline, or venlafaxine). 10,11

CONCLUSION: All Patients who are on neuroleptic medication and especially with risk factors for developing NMS should be monitored carefully to prevent life threatening complication of NMS.

In our case patient recovered promptly due to early diagnoses and prompt treatment with dopamine agonist bromocriptine, withdrawal of antipsychotic and good supportive care.

\section{REFERENCES:}

1. Levenson J L. Neuroleptic malignant syndrome. Am J Psychiatry 1985; 142:1137-1145.

2. Bhanushali $\mathrm{M} \mathrm{J}$, Tuite $\mathrm{P} J$. The evaluation and management of patient's with neuroleptic malignant syndrome. Neurol Clin 2004; 22:389-411.

3. American Psychiatric Association. Diagnostic and statistical Manual, Fourth Edition (Text Revision) 2001.

4. Velamoor VR. Neuroleptic malignant syndrome: recognition, prevention and management. Drug Saf. 1998; 19:73-82.

5. Caroff S, Mann S, Campbell E. Atypical antipsychotics and neuroleptic malignant syndrome. Psych Annals 2000; 30:314-321

6. Sachdev P, Mason C, Hadzi-Pavlovic D. Case-control study of neuroleptic malignant syndrome. Am J Psychiatry 1997; 154:1156-1159

7. Pelonero AL, Levensons JL, Silverman JJ. Neuroleptic therapy following Neuroleptic malignant syndrome. Psychosomatics 1985; 26:946-947.

8. U.S. Food and Drug Administration. Medwatch online. Available at: http://www.fda.gov/medwatch/SAFETY/2004/abilify_pi.pdf. Accessed June 7, 2004.

9. Nakai S, Hirose T, Uwahodo Y, et al. Diminished catalepsy and dopamine metabolism distinguish aripiprazole from haloperidol or risperidone. Eur J Pharmacol 2003; 472:89-97. 
10. DeQuardo J. Worsened agitation with aripiprazole: adverse effect of dopamine partial agonism? J Clin Psychiatry 2004; 65:132-3.

11. Cohen S, Rulf D, Pies R. Extrapyramidal side effects associated with aripiprazole coprescription in 2 patients. J Clin Psychiatry 2005; 66:135-6.

\section{AUTHORS:}

1. Nayana Naik

2. Yvonne Da Silva Pereira,

3. Ashish Srivastava

\section{PARTICULARS OF CONTRIBUTORS:}

1. Assistant Professor, Department of Psychiatry, Institute of Psychiatry and Human Behavior [IPHB], Bambolim, Goa.

2. Professor and HOD, Department of Psychiatry, Institute of Psychiatry and Human Behavior [IPHB], Bambolim, Goa.

3. Lecturer, Department of Psychiatry, Institute of Psychiatry and Human Behavior [IPHB], Bambolim, Goa.

\section{NAME ADDRESS EMAIL ID OF THE} CORRESPONDING AUTHOR:

Dr. Nayana Naik,

Bf 41, "OM-HARI", Housing Complex, Goa Housing Board, Alto Porvorim Bardez, Goa, PIN - 403521.

Email- nnaik2002@yahoo.co.in

Date of Submission: 09/08/2013.

Date of Peer Review: 10/08/2013.

Date of Acceptance: 11/08/2013.

Date of Publishing: 16/08/2013 